\title{
COPAR: A ChIP-Seq Optimal Peak Analyzer
}

\author{
Binhua Tang, ${ }^{1,2}$ Xihan Wang, ${ }^{1}$ and Victor X. Jin ${ }^{3}$ \\ ${ }^{1}$ Epigenetics \& Function Group, School of Internet of Things, Hohai University, Jiangsu 213022, China \\ ${ }^{2}$ School of Public Health \& Biostatistics, Shanghai Jiao Tong University, Shanghai 200025, China \\ ${ }^{3}$ Department of Molecular Medicine \& Biostatistics, University of Texas Health Science Center, San Antonio, TX 78249, USA
}

Correspondence should be addressed to Binhua Tang; bh.tang@outlook.com

Received 28 October 2016; Accepted 14 February 2017; Published 5 March 2017

Academic Editor: Xingming Zhao

Copyright (C) 2017 Binhua Tang et al. This is an open access article distributed under the Creative Commons Attribution License, which permits unrestricted use, distribution, and reproduction in any medium, provided the original work is properly cited.

\begin{abstract}
Sequencing data quality and peak alignment efficiency of ChIP-sequencing profiles are directly related to the reliability and reproducibility of NGS experiments. Till now, there is no tool specifically designed for optimal peak alignment estimation and quality-related genomic feature extraction for ChIP-sequencing profiles. We developed open-sourced COPAR, a user-friendly package, to statistically investigate, quantify, and visualize the optimal peak alignment and inherent genomic features using ChIPseq data from NGS experiments. It provides a versatile perspective for biologists to perform quality-check for high-throughput experiments and optimize their experiment design. The package COPAR can process mapped ChIP-seq read file in BED format and output statistically sound results for multiple high-throughput experiments. Together with three public ChIP-seq data sets verified with the developed package, we have deposited COPAR on GitHub under a GNU GPL license.
\end{abstract}

\section{Introduction}

Next-generation sequencing (NGS) integrated with ChIP technology provides a genome-wide perspective for biomedical research and clinical diagnosis applications [1-3].

Data quality and peak alignment of ChIP-sequencing profiles are directly related to the reliability and reproducibility of analysis results. For example, ChIP-seq data characterize alteration evidence for transcription factor (TF) binding activities in response to chemical or environmental stimuli, but if the ChIP-seq alignment is poorly selected, any follow-up analysis may lead to inaccurate TF binding results and inevitable loss of biological meanings $[4,5]$.

The mostly investigated items in ChIP-seq peak calling procedures are peak number, false discovery rate (FDR), corresponding bin-size, and other statistical thresholds selected in each analysis. Without exception, such arguments form impenetrable barriers for biologists and bioinformaticians to choose a suitable pair condition for analyzing experimental results.

And to our knowledge, few literatures or application notes focus on such topics; thus herein we propose a flexible package based on feature extraction and signal processing algorithms for solving such an argument-selection optimization problem in optimal peak alignment.

In summary, the package COPAR can quantitatively measure NGS/ChIP-seq experiment quality through global peak alignment comparison and extract genomic features based on spectrum method for in-depth analysis of ChIPsequencing profiles.

\section{Materials and Methods}

2.1. Optimal Peak Alignment Estimation. For determining optimal ChIP-seq alignment, we need to analyze peak numbers under specific argument constraints. Thus we acquire optimal peak numbers by constraining specific arguments, which can be formalized as a class of optimal track analysis, illustrated as

$$
\begin{array}{cl}
\underset{i}{\arg \max } & P_{i}, \quad i \in N \\
\text { s.t. } & f_{i} \leq \chi, \\
& b_{i}=\beta, \\
& p_{i} \leq \delta,
\end{array}
$$




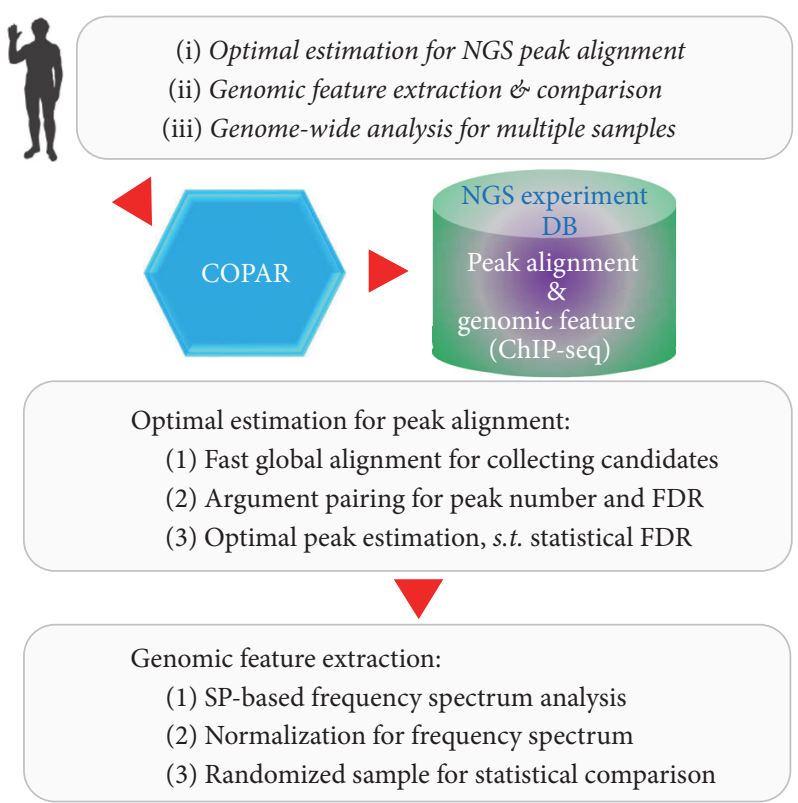

FIGURE 1: Flowchart for optimal peak alignment estimation and genomic feature analysis with COPAR. The package can perform optimal peak estimation based on global alignment of ChIP-seq data; then it can utilize the frequency spectrum approach for genomic feature extraction and carries out statistical comparison for multiple ChIP-seq samples.

where $P_{i}$ denotes a set of optimal peak numbers under corresponding argument constraints, $f_{i}$ stands for argument FDR, $b_{i}$ stands for bin-size, $p_{i}$ denotes $p$ value threshold, and $\chi, \beta$, and $\delta$ represent the presupposed argument values, respectively.

2.2. Spectrum-Based Genomic Feature Extraction. For a finite random variable sequence, its power spectrum is normally estimated from its autocorrelation sequence by use of discrete-time Fourier transform (DTFT), denoted as [6-8]

$$
P(\omega)=\frac{1}{2 \pi} \sum_{n=-\infty}^{\infty} C_{x x}(n) e^{-j n \omega},
$$

where $C_{x x}$ denotes autocorrelation sequence of a discrete signal $x_{n}$, defined as

$$
C_{x x}(i, j)=\frac{E\left[\left(X_{i}-\mu_{i}\right)\left(X_{j}-\mu_{j}\right)\right]}{\sigma_{i} \sigma_{j}},
$$

where $\mu$ and $\sigma$ stand for mean and variance, respectively.

In our study, for consideration of the ChIP-seq data characteristics, we use 128 sampling points to calculate discrete Fourier transform, with the related sampling frequency $1 \mathrm{KHz}$.

\section{Results}

The COPAR package was developed and open-sourced for academic biologists, and it uses built-in functions for determining optimal peak alignment candidate and extracting genomic features from ChIP-seq dataset.
The package is designed to handle BED-formatted ChIPseq data as input [9], and it can process single ChIP-seq for optimal peak alignment and feature extraction analysis, together with the capability to perform genome-wide statistical comparison for multiple ChIP-seq samples. The analysis flowchart for the package is given in Figure 1.

It can automatically determine the optimal peak alignment with statistically meaningful FDR through fast global alignment comparison; the global comparison is subject to two statistical arguments, namely, bin-size and $p$ value threshold.

The functionalities of our developed package are largely complementary to and extend current tools used for ChIPseq data analysis. The optimal peak alignment estimation is shown in Figures 2(a) and 2(b); and the spectrum-based feature extraction is given in Figures 2(c) and 2(d). Figures 2(a) and 2(b) utilize heatmap to represent peak number and corresponding FDR candidate subject to each argument pair, binsize (vertical axis), and $p$ value threshold (horizontal axis), respectively; Figure 2(c) denotes the spectrum distribution of the global peak alignment candidate sequence, normalized with its frequency range $[0,500] \mathrm{Hz}$ and magnitude within $[-40,-3] \mathrm{dB}$; Figure 2(d) denotes the randomized case.

\section{Conclusions}

Based on global peak alignment, COPAR optimizes the argument selection in ChIP-seq analysis; meanwhile, COPAR utilizes the signal spectrum processing method to further extract genomic features and statistically compare multiple ChIP-seq samples for NGS high-throughput experiments.

In summary, our developed package COPAR can process mapped read file in BED format and output statistically sound 


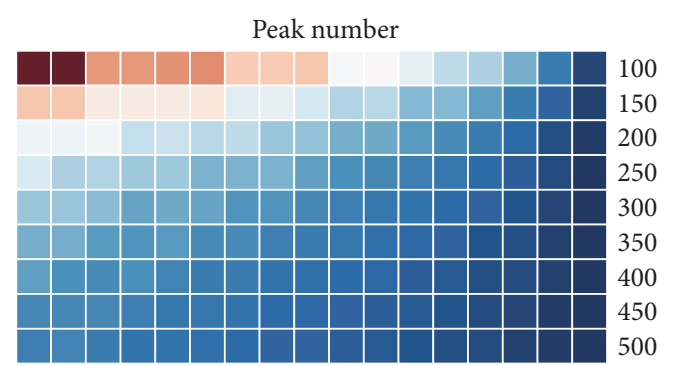

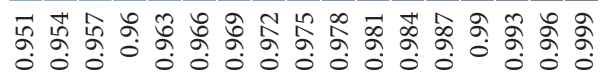

(a)

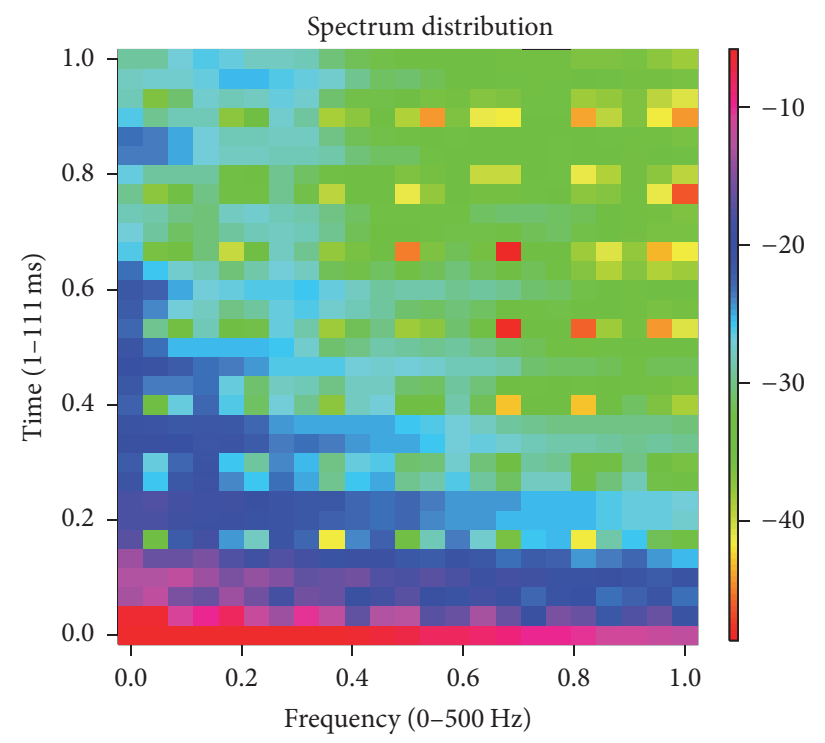

(c)

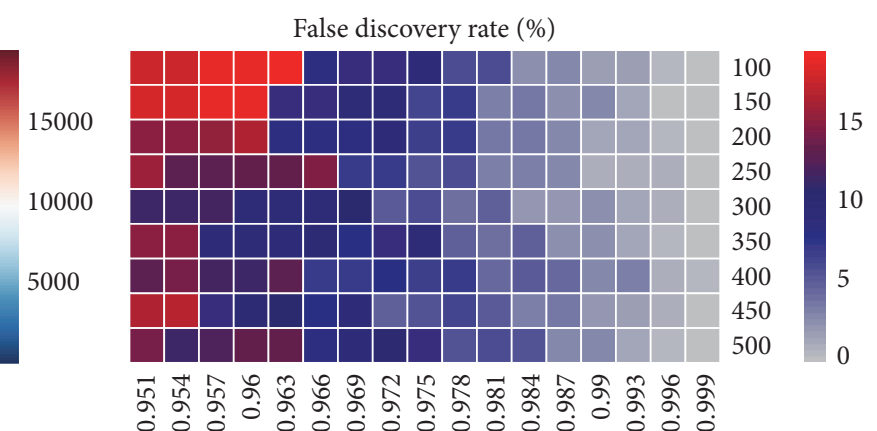

(b)

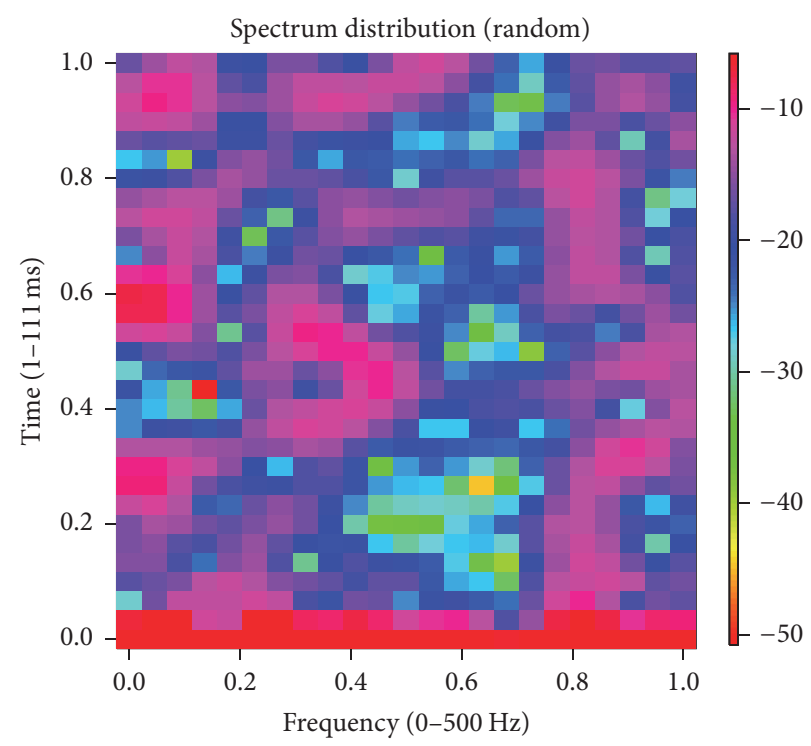

(d)

FIGURE 2: Global optimal peak analysis result subject to the arguments bin-size and FDR. (a) Global distributions for peak number candidates and (b) corresponding false discovery rate, subject to bin-size (vertical axis, from 100 through $500 \mathrm{bp}$ ) and $p$ value threshold (horizontal axis, from 0.951 to 0.999 ), respectively; (c) genomic feature extraction based on spectrum distribution for global peak number candidates identified from COPAR; (d) spectrum distribution for the randomized sequence.

results for diverse high-throughput sequencing experiments; we further verified the package with three GEO ChIP-seq datasets as study cases, and we included the analysis results into the package manual. The developed package COPAR is currently available under a GNU GPL license from https:// github.com/gladex/COPAR.

\section{Abbreviations \\ NGS: $\quad$ Next-generation sequencing \\ ChIP-seq: Chromatin immunoprecipitation-sequencing \\ FDR: False discovery rate \\ TF: Transcription factor \\ DTFT: Discrete-time Fourier transform.}

\section{Competing Interests}

The authors declare that they have no competing interests.

\section{Authors' Contributions}

Binhua Tang and Victor X. Jin conceived the method; Binhua Tang and Xihan Wang wrote and compiled the package; Binhua Tang, Xihan Wang, and Victor X. Jin drafted and proof-checked the manuscript.

\section{Acknowledgments}

This work has been supported by the Natural Science Foundation of Jiangsu, China (BE2016655 and BK20161196), Fundamental Research Funds for China Central Universities (2016B08914), and Changzhou Science \& Technology Program (CE20155050). This work made use of the resources supported by the NSFC-Guangdong Mutual Funds for Super Computing Program (2nd Phase) and the Open Cloud Consortium- (OCC-) sponsored project resource, supported in part by grants from Gordon and Betty Moore Foundation 
and the National Science Foundation (USA) and major contributions from OCC members.

\section{References}

[1] E. R. Mardis, "ChIP-seq: welcome to the new frontier," Nature Methods, vol. 4, no. 8, pp. 613-614, 2007.

[2] G. J. Martinez and A. Rao, "Cooperative transcription factor complexes in control," Science, vol. 338, no. 6109, pp. 891-892, 2012.

[3] H. Kilpinen and J. C. Barrett, "How next-generation sequencing is transforming complex disease genetics," Trends in Genetics, vol. 29, no. 1, pp. 23-30, 2013.

[4] M. D. Chikina and O. G. Troyanskaya, "An effective statistical evaluation of chipseq dataset similarity," Bioinformatics, vol. 28, no. 5, pp. 607-613, 2012.

[5] T. S. Furey, "ChIP-seq and beyond: new and improved methodologies to detect and characterize protein-DNA interactions," Nature Reviews Genetics, vol. 13, no. 12, pp. 840-852, 2012.

[6] A. V. Oppenheim and R. W. Schafer, Discrete-Time Signal Processing, Prentice Hall, Upper Saddle River, NJ, USA, 3rd edition, 2010.

[7] B. Tang, H.-K. Hsu, P.-Y. Hsu et al., "Hierarchical modularity in ER $\alpha$ transcriptional network is associated with distinct functions and implicates clinical outcomes," Scientific Reports, vol. 2, article 875, 2012.

[8] S.-L. Wang, Y.-H. Zhu, W. Jia, and D.-S. Huang, "Robust classification method of tumor subtype by using correlation filters," IEEE/ACM Transactions on Computational Biology and Bioinformatics, vol. 9, no. 2, pp. 580-591, 2012.

[9] X. Lan, R. Bonneville, J. Apostolos, W. Wu, and V. X. Jin, "WChIPeaks: a comprehensive web application tool for processing ChIP-chip and ChIP-seq data," Bioinformatics, vol. 27, no. 3, pp. 428-430, 2011. 

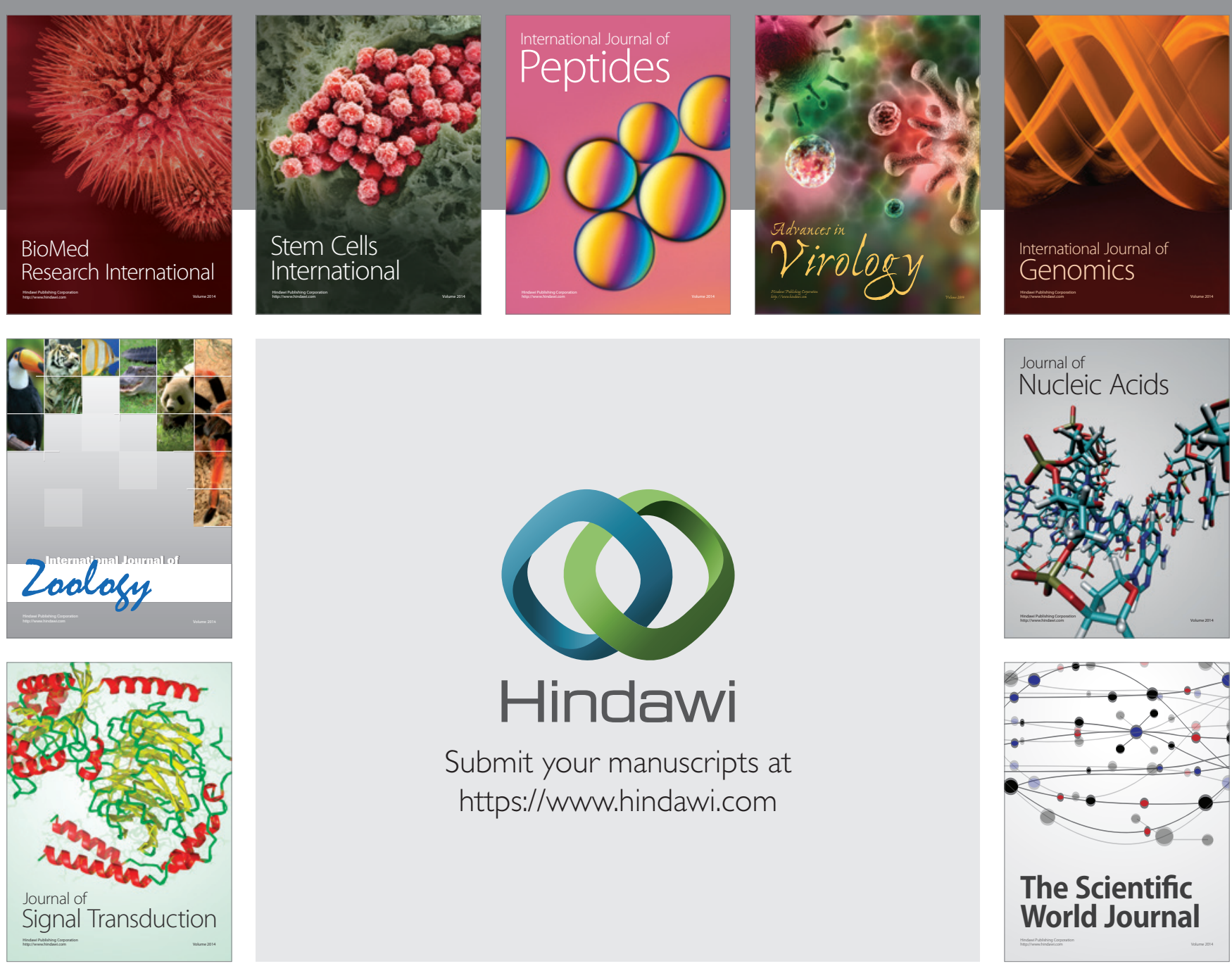

Submit your manuscripts at

https://www.hindawi.com
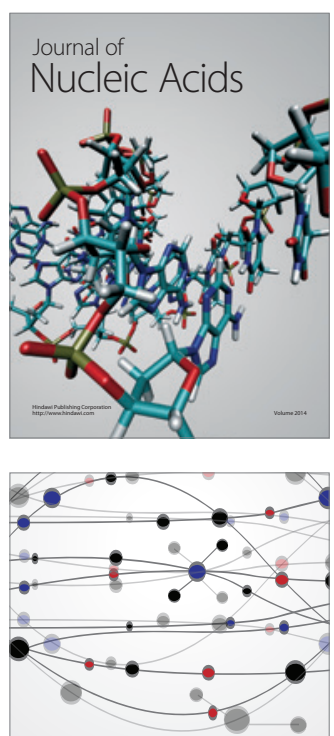

The Scientific World Journal
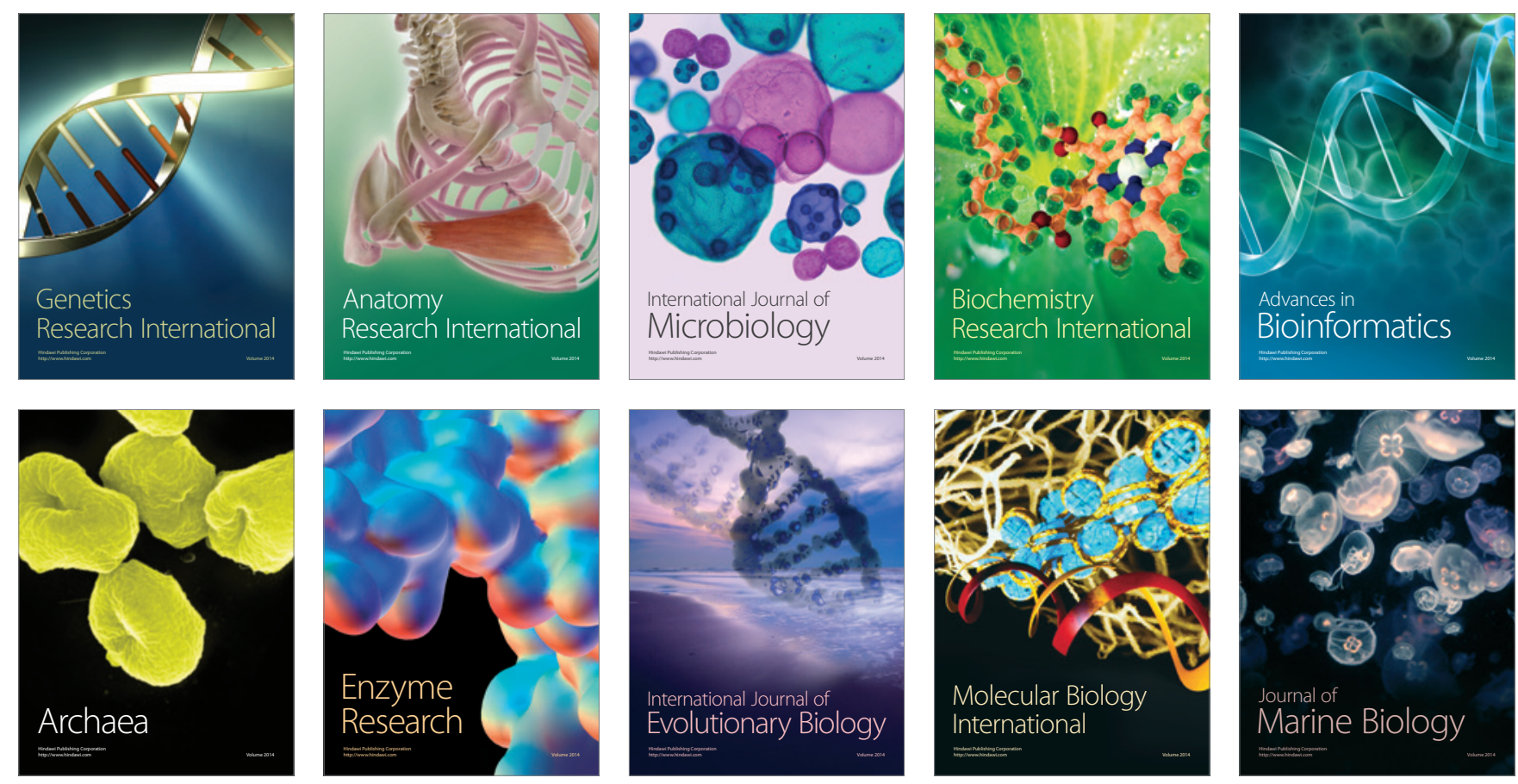